\title{
Targeting liver stage malaria with metformin
}

\author{
Iset Medina Vera, ${ }^{1}$ Margarida T. Grilo Ruivo,' Leonardo F. Lemos Rocha,' Sofia Marques, ${ }^{1}$ \\ Sangeeta N. Bhatia, ${ }^{2,3,4}$ Maria M. Mota, ${ }^{1}$ and Liliana Mancio-Silva ${ }^{1,2,3}$ \\ IInstituto de Medicina Molecular João Lobo Antunes, Faculdade de Medicina Universidade de Lisboa, Lisboa, Portugal. \\ ${ }^{2}$ Institute for Medical Engineering and Science, Massachusetts Institute of Technology, Cambridge, Massachusetts, USA \\ ${ }^{3}$ Koch Institute for Integrative Cancer Research, Cambridge, Massachusetts, USA. ${ }^{4}$ Howard Hughes Medical Institute, \\ Cambridge, Masschusetts, USA.
}

\begin{abstract}
Despite an unprecedented 2 decades of success, the combat against malaria - the mosquitotransmitted disease caused by Plasmodium parasites - is no longer progressing. Efforts toward eradication are threatened by the lack of an effective vaccine and a rise in antiparasite drug resistance. Alternative approaches are urgently needed. Repurposing of available, approved drugs with distinct modes of action are being considered as viable and immediate adjuncts to standard antimicrobial treatment. Such strategies may be well suited to the obligatory and clinically silent first phase of Plasmodium infection, where massive parasite replication occurs within hepatocytes in the liver. Here, we report that the widely used antidiabetic drug, metformin, impairs parasite liver stage development of both rodent-infecting Plasmodium berghei and human-infecting $P$. falciparum parasites. Prophylactic treatment with metformin curtails parasite intracellular growth in vitro. An additional effect was observed in mice with a decrease in the numbers of infected hepatocytes. Moreover, metformin provided in combination with conventional liver- or bloodacting antimalarial drugs further reduced the total burden of $P$. berghei infection and substantially lessened disease severity in mice. Together, our findings indicate that repurposing of metformin in a prophylactic regimen could be considered for malaria chemoprevention.
\end{abstract}

Conflict of interest: The authors have declared that no conflict of interest exists.

Copyright: (c) 2019, American Society for Clinical Investigation.

Submitted: January 18, 2019 Accepted: November 13, 2019 Published: December 19, 2019

Reference information: JCI Insight. 2019;4(24):e127441. https://doi.org/10.1172/jci. insight.127441.

\section{Introduction}

Malaria continues to be a global health problem, limiting economic growth and progress in high-burden communities. Nearly half of the world's population is at risk for infection, with 219 million new cases reported in 2017, leading to nearly half a million deaths per year. Malaria is caused by intracellular parasites of the genus Plasmodium; of these, Plasmodium falciparum is the most virulent form among the 5 species (P. vivax, P. ovale, P. malaria, and $P$. knowlesi) that infect humans (1). Malaria infection begins in the liver, an obligatory step where mosquito-delivered Plasmodium sporozoites invade hepatocytes and replicate within a parasitophorous vacuole. This affords the parasite the protection and resources to multiply from 1 initial infectious parasite into thousands of daughter merozoites. These are then released to the bloodstream and go on to infect erythrocytes, commencing the symptomatic, cyclic, and pathogenic blood stage of infection (2). Although clinically silent, the liver stage of infection is a research priority because it is the first stage for stopping infection before fulminant disease. The liver stage of malaria infection is also important for development of sterile immunity and holds promise for vaccine development (3).

Traditional antimalarial drug discovery pipelines have been designed to target the Plasmodium parasite (4). However, current antimalarial interventions based on this approach have shortcomings, such as (a) restricted number of effective drugs, (b) adverse side effects and restricted use, (c) development of drug resistance, and (d) limited mechanisms of action. For example, primaquine and tafenoquine, 8-aminoquinolines that target malaria liver stages, are the only drugs approved to treat $P$. vivax hypnozoites - the long-lived, dormant, hepatic forms that cause malaria relapses. However, their short half-life, long dosage regimens, and incompatibility with glucose-6-phosphate dehydrogenase deficiency (which requires prescreening of recipients) makes them unsuitable for widespread use (5). Despite recent advances in novel parasite-directed antimalarials, which hold much promise and are currently in the clinical pipeline, there is still a need for alternative approaches (6). Chemotherapy that targets the host, or both the host and the parasite, is a reasonable, complementary, and alternative approach to combat malaria because it would address several of the shortcomings of current treatments, for example, by circumventing potential for generation of drug-resistant parasites (7-10). 
A

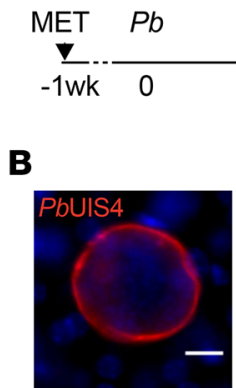

CTL

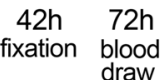
draw

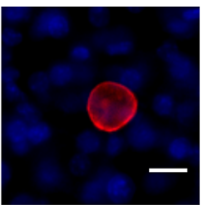

MET

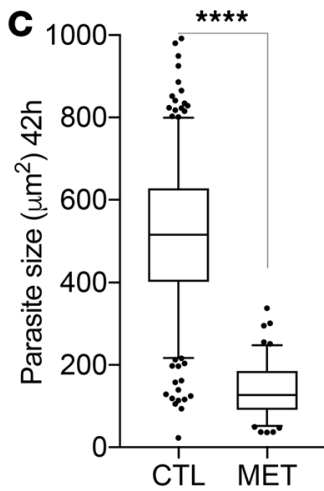

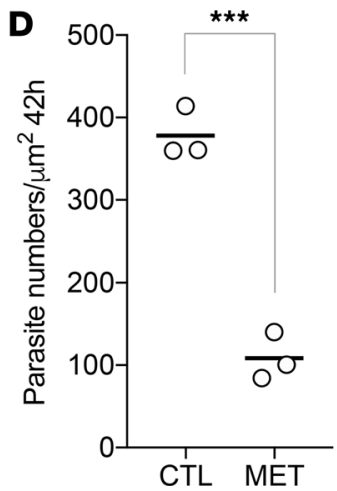

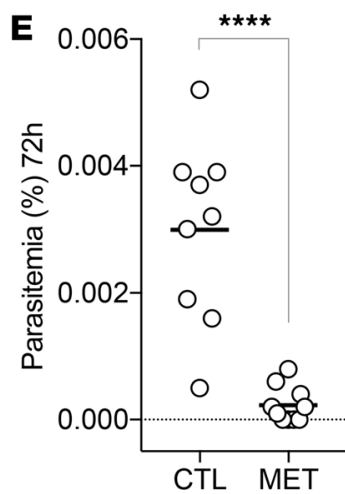

Figure 1. Metformin treatment inhibits $\boldsymbol{P}$. berghei liver infection in mice. (A) Timeline of drug treatment, infection, and sample collection. C57BL/6 mice were injected intravenously with GFP-expressing $P$. berghei sporozoites (10,000 inoculum for B-D, 500 inoculum for E). Metformin (MET, $500 \mathrm{mg} / \mathrm{kg} / \mathrm{d}$ ) was given prophylactically in the drinking water 1 week before and during infection. Nontreated control (CTL) received regular water. Livers were harvested at 42 hours and tail blood was collected 72 hours after infection. (B) Representative immunofluorescence images of $P$. berghei schizonts in liver sections at 42 hours after infection. Parasites were visualized using anti-GFP (not shown) to detect the reporter transgene and anti-PbUIS4, a parasitophorous membrane marker (shown in red). Nuclei were stained with Hoechst (shown in blue). Scale bar: $10 \mu \mathrm{m}$. (C) Box plot of $P$. berghei size distribution in liver sections at 42 hours. The total number of parasites analyzed in 3 infected mice per group is 377, CTL; and 115, MET. The outliers in the box plots represent $5 \%$ of data points. The box plots depict the minimum and maximum values (whiskers), the upper and lower quartiles, and the median. The length of the box represents the interquartile range. Mann-Whitney $U$ test, ${ }^{* * *} P<0.0001$. (D) Scatter plot of parasite density per square micrometer of infected liver sections at 42 hours. Each dot represents one animal and the horizontal bar represents the mean. Student's $t$ test, ${ }^{* * *} P<0.001$. (E) Scatter plot showing the percentage of infected erythrocytes (parasitemia) measured by flow cytometry 72 hours after infection. Each dot represents 1 animal and the horizontal bar represents the mean. The total number of mice analyzed in 2 independent experiments is 9, CTL; and 10, MET. Mann-Whitney $U$ test, ${ }^{* * * * P<0.0001 .}$

These approaches may lead to indirect effects on parasite replication or development through targeting of processes essential for parasitism. Such mechanisms include limiting nutrient acquisition by the parasite or overcoming pathogen-imposed blocks in immune recognition and activation of cell-intrinsic defense mechanisms, such as autophagy. Additionally, adjunct-directed therapy might help reduce collateral effects from infection, such as excessive inflammation leading to tissue damage. Additionally, adjunct-directed therapy may promote immune competence for effective parasite clearance and activation of immune memory (7). Alternative targets that have been proposed as antimalarial interventions include erythrocyte $G$ protein (11), heme oxygenase I $(12,13), \operatorname{CD} 81$ and SRBI $(14,15)$, p53 and Bcl2 (16, 17), and several classes of host kinases (18-20). Among these is the key regulator of cell energy homeostasis, AMP kinase (AMPK) (20). In a previous work, we demonstrated that AMPK negatively modulates hepatic infection by Plasmodium parasites and that several AMPK-activating drugs cause a reduction in the intracellular growth of rodent $P$. berghei parasites in vitro (20). In this study, we report on the in vivo antimalarial effect of metformin, a known AMPK agonist widely used as an antidiabetic drug. We evaluated its potential use as a prophylactic and adjunctive treatment in combination with clinically approved antimalarials.

\section{Results}

Metformin treatment impairs P.berghei liver infection in mice. Metformin is the first-line treatment for type 2 diabetes; it primarily targets the liver, where it acts to lower systemic blood glucose levels. In the liver, metformin can reach remarkably high levels with reported concentrations ranging between $60 \mu \mathrm{M}(250 \mathrm{mg} /$ $\mathrm{kg}$ administered by drinking water) to $1 \mathrm{mM}(350 \mathrm{mg} / \mathrm{kg}$ by oral gavage). This is in contrast with the $5 \mu \mathrm{M}$ found in the plasma of those animals (21). Metformin accumulation in the liver is due to the high expression of organic cation transporters (OCTs) in the hepatocyte plasma membrane enabling drug import. This contributes to efficient reduction of hepatic glucose production and output (22). Unlike hepatocytes, hepatoma cancer cells in vitro downregulate OCT expression $(23,24)$, which might affect effective drug dosing. Because our previous work was performed in a Huh7 hepatoma cell line (20), we decided to revisit the data and probe the activity of metformin in freshly isolated primary mouse hepatocytes infected with rodent $P$. berghei sporozoites. We observed a 3 -fold reduction of the $50 \%$ inhibitory concentration $\left(\mathrm{IC}_{50}\right)$ required to impair the parasite intracellular development in primary hepatocytes compared with hepatoma cells (45.17 $\pm 0.05 \mu \mathrm{M}$ vs. $156 \pm 70 \mu \mathrm{M}$, respectively; Supplemental Figure 1; supplemental material available online with this article; https://doi.org/10.1172/jci.insight.127441DS1). This result prompted us to evaluate the in vivo efficacy of metformin in a mouse model of acute malaria. Metformin was administered to mice 
A

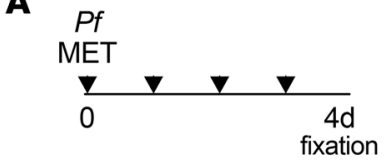

B

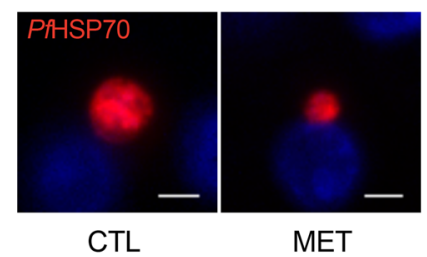

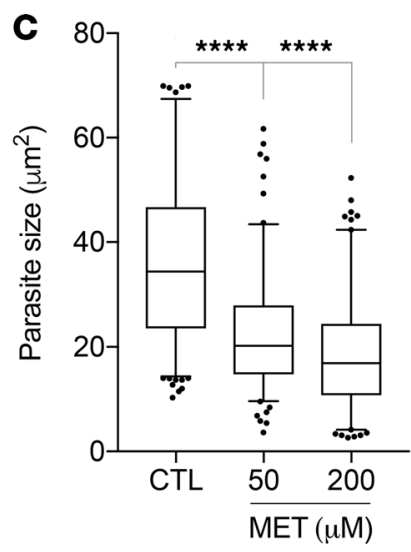

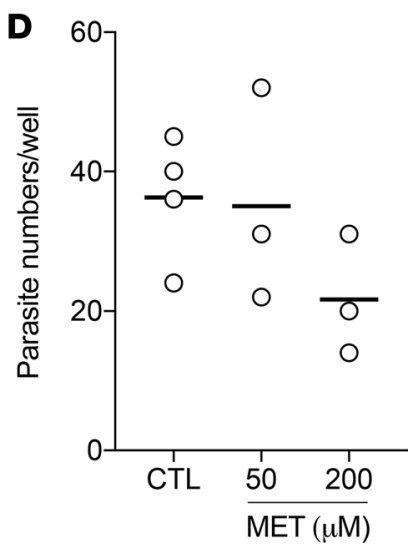

Figure 2. Metformin treatment reduces $\boldsymbol{P}$. falciparum development in human hepatocytes. (A) Timeline of metformin treatment, infection, and sample collection. Primary human hepatocytes were infected with $60,000 P$. falciparum sporozoites. Prophylactic dosing of metformin (50 and $200 \mu \mathrm{M})$ started at 3 hours after infection and repeated daily. Cultures were fixed at day 4 after infection. (B) Representative immunofluorescence images of $P$. falciparum parasites stained with anti-PfHSP70 antibodies (shown in red) for nontreated control and $200 \mu \mathrm{M}$ metformin treatment. Nuclei were stained with Hoechst (shown in blue). Scale bar: $5 \mu \mathrm{m}$. (C and $\mathbf{D}$ ) Quantification of $P$. falciparum size distribution (C) and density (D) at day 4 after infection. The total number of parasites analyzed in 2 independent experiments is 190, CTL; 143, MET $50 \mu \mathrm{M} ; 140$, MET $200 \mu \mathrm{M}$. The outliers in the box plots represent $5 \%$ of data points. The box plots depict the minimum and maximum values (whiskers), the upper and lower quartiles, and the median. The length of the box represents the interquartile range. The dots in the scatter plot represent 1 well, and the horizontal bars show the mean, in 1 of the 2 experiments. Ordinary 1-way ANOVA test, ${ }^{* * *} P<0.0001$.

orally via drinking water at approximately $500 \mathrm{mg} / \mathrm{kg} / \mathrm{d}$, a dose previously validated in mice infected with Mycobacterium tuberculosis (25). Metformin treatment over a week resulted in plasma concentrations of 3-7 $\mu \mathrm{M}$, which is comparable to levels previously reported in mice (21) and diabetic patients under treatment (26). No differences in water intake and body weight were observed between the treated and nontreated animals (Supplemental Figure 2). Mice treated for a week were then infected by intravenous injection of $P$. berghei sporozoites (Figure 1A). The livers of infected mice were harvested 42 hours after infection, sectioned, and analyzed by fluorescence microscopy using parasite-specific markers. Consistent with our previous in vitro data (20), we observed that individual hepatic schizonts in metformin-treated mice were dramatically smaller than those in nontreated mice (492 $\pm 167 \mu \mathrm{m}^{2}$ vs. $140 \pm 63 \mu \mathrm{m}^{2} ; P<0.0001$; Figure 1 , $B$ and $C)$. Additionally, microscopy analysis revealed that the number of infected hepatocytes was significantly reduced in metformin-treated relative to the nontreated control mice $\left(378 \pm 31\right.$ parasites $/ \mu \mathrm{m}^{2} \mathrm{vs} .108$ \pm 29 parasites $/ \mu \mathrm{m}^{2} ; P<0.001$; Figure 1D). To assess the global impact of metformin treatment, we infected mice with 500 sporozoites, an inoculum that is expected to mimic a natural infection by a mosquito bite $(27,28)$, and allowed the entire liver stage development to be completed. We then determined the prepatent parasitemia by flow cytometry analysis 72 hours after infection. Strikingly, while the entire group of nontreated mice presented with parasites in the blood, we were unable to detect $P$. berghei-infected erythrocytes in $40 \%$ of the metformin-treated mice (Figure 1E). The remaining $60 \%$ of mice showed an $87 \%$ reduction in parasitemia levels relative to the nontreated control $(P<0.0001$; Figure 1E). These results show that metformin has a potent activity against liver stage malaria in vivo.

Metformin treatment reduces $P$. falciparum growth in human hepatocytes. Next, we sought to validate our findings in a more clinically relevant $P$. falciparum infection model. Cryopreserved primary human hepatocytes were maintained in micropatterned cocultures in vitro containing supportive fibroblasts (29-32), infected with P. falciparum sporozoites, and treated daily with metformin (Figure 2A). Consistent with the $P$. berghei experiments, metformin treatment reduced the intracellular development of $P$. falciparum parasites, in a dose-dependent manner, as seen by a significant decrease in the mean size of hepatic schizonts at day 4 after sporozoite infection $(P<0.0001$; Figure $2, \mathrm{~B}$ and $\mathrm{C})$. Unlike the data obtained in mice, however, we did not observe significant differences in the number of infected hepatocytes (Figure 2D). This discrepancy might be due to antiinflammatory alterations promoted by metformin in vivo $(25,33)$, which might lead to efficient clearance of liver stage Plasmodium parasites, or a defect in parasite hepatocyte invasion due to pretreatment. 
A

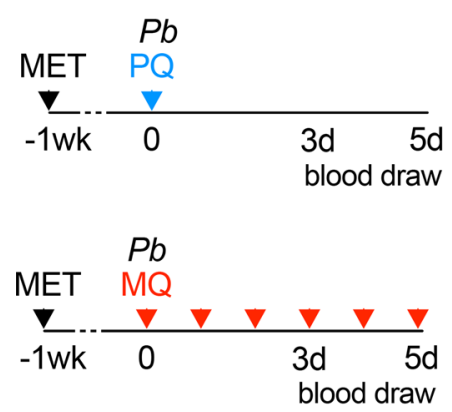

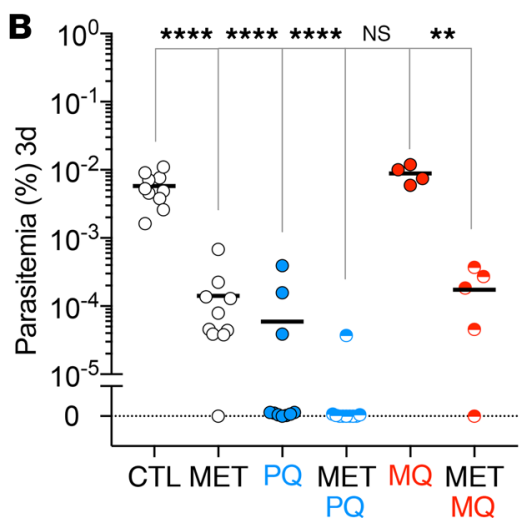

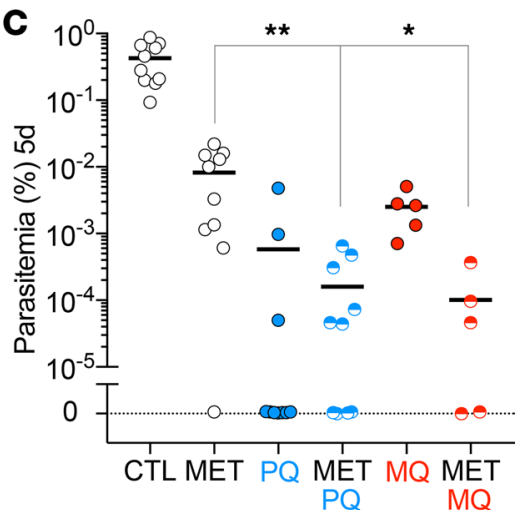

Figure 3. Combined metformin treatment with suboptimal doses of primaquine or mefloquine improves antimalarial effect. (A) Timeline of drug treatments, infections, and sample collection. C57BL/6 mice were injected intravenously with $500 \mathrm{GFP}$-expressing $P$. berghei sporozoites. Metformin (500 mg/ $\mathrm{kg} / \mathrm{d}$ ) was provided in the drinking water 1 week before and during infection. Primaquine (PQ, $15 \mathrm{mg} / \mathrm{kg}$ ) was injected 1 time intraperitoneally 2 hours after infection. Mefloquine (MQ, $10 \mathrm{mg} / \mathrm{kg}$ ) was given daily by intraperitoneal injection. (B and C) Scatter plot showing the percentage of infected erythrocytes (parasitemia) measured by flow cytometry at day 3 ( 72 hours, B) and day 5 (C) after infection. Each dot represents 1 animal. The horizontal bar represents the mean. The total number of mice analyzed is as follows: 10, CTL; 10, MET; 10, PQ; 10, MET/PQ; 5, MQ; and 5, MET/MQ. P values calculated relative to nontreated CTL (B) and relative to MET (C). Ordinary 1-way ANOVA test, ${ }^{* * * *} P<0.0001 ;{ }^{* *} P<0.01 ;{ }^{*} P<0.05$; ns, not significant.

Metformin treatment is inefficient in Plasmodium asexual erythrocytic stages. To evaluate the curative potential of metformin on symptomatic blood stage infection, we determined dose-response curves in $P$. falciparum blood cultures and monitored the parasitemia in treated and nontreated mice infected with $P$. berghei sporozoites. For the in vitro sensitivity studies, synchronized blood cultures of P. falciparum were exposed to escalating doses of metformin and compared with the nontreated condition. Parasite growth inhibition curves determined after a 48-hour incubation showed an $\mathrm{IC}_{50}$ value considerably higher than that of liver stages in vitro $(3.7 \pm 1.1 \mathrm{mM}$ vs. $45.17 \pm 0.05 \mu \mathrm{M}$, respectively; Supplemental Figure 3A). In mice infected with a natural inoculum of sporozoites (as above), we observed similar growth in the linear phase of replication (days 3-5 of patent blood stage infection), with no significant difference in the calculated parasite multiplication rate between nontreated and metformin-treated animals (Supplemental Figure 3B). Collectively, our results suggest a weak effect of metformin on infected erythrocytes, which is in agreement with historical data reporting no effect in patients with an ongoing $P$. vivax blood stage infection (34). Furthermore, a recent study showed similar parasitemia in nontreated and metformin-treated mice infected with $P$. yoelii (35).

Metformin adjunctive treatment enhances effect of antimalarial drugs. Given the robust inhibitory effect of metformin treatment on both $P$. berghei and $P$. falciparum infections in the liver, we next explored whether combination with suboptimal doses of conventional antimalarial drugs that are more likely to result in fewer side effects could improve the effectiveness of the prophylactic treatment. First, we tested a combination of metformin with primaquine, a drug targeting liver stage parasites. Mice were pretreated with metformin in drinking water and infected with $500 \mathrm{P}$. berghei sporozoites (as above). A single suboptimal dose of primaquine was then administered via intraperitoneal injection to nontreated and metformin-treated mice. The end result of liver infection was detected 72 hours after infection by assessing the number of infected erythrocytes by flow cytometry (Figure 3A). Although the entire group of nontreated mice exhibited prepatent parasitemia, the combined treatment of metformin and primaquine resulted in a remarkable inhibition of liver infection, with only $10 \%$ of mice showing parasites in blood (Figure 3B). The outcome of the single-drug treatments was $30 \%$ and $90 \%$ blood positive for primaquine and metformin, respectively (Figure 3B and Table 1).

Next, we evaluated the combined effect of metformin with a suboptimal dosage of mefloquine, one of the most used drugs for antimalarial prevention in travelers, despite the numerous side effects, such as headaches, dizziness, insomnia, and psychological disturbances (36). Unlike primaquine, mefloquine is active against blood stages of malaria parasites but not liver stages. Mefloquine was administered by daily intraperitoneal injections starting on the day of infection with sporozoites and was continued up to day 5 (Figure 3A). As expected, the single or combined mefloquine treatment did not affect the total parasite load measured at 72 hours when compared to nontreated mice or metformin treatment alone (Figure 3B). As the parasites continued proliferating in the blood, mefloquine inhibitory activity became evident. Specifically, we observed that parasitemia was significantly lower in mice with the combined treatment relative to mice 
Table 1. Prepatency and survival of nontreated and treated mice

\begin{tabular}{|c|c|c|c|}
\hline & \multicolumn{2}{|c|}{ Prepatency } & \multirow{2}{*}{$\begin{array}{c}\text { Survival } \\
\text { Day } 10\end{array}$} \\
\hline & Day 3 & Day 5 & \\
\hline No treatment & 19 (19) & 19 (19) & 3 (19) \\
\hline Metformin & $17(20)$ & $19(20)$ & $16(20)$ \\
\hline Primaquine & 3 (10) & 3 (10) & $10(10)$ \\
\hline Metformin/Primaquine & $1(10)$ & $6(10)$ & $10(10)$ \\
\hline Mefloquine & $5(5)$ & $5(5)$ & $5(5)$ \\
\hline Metformin/Mefloquine & $4(5)$ & $3(5)$ & $5(5)$ \\
\hline \multicolumn{4}{|c|}{$\begin{array}{l}\text { Summary of C57BL/ } 6 \text { mice infected with GFP-expressing } P \text {. berghei sporozoites and treated with metformin, } \\
\text { primaquine, and mefloquine, alone or in combination as described in Figures } 1 \text { and } 3 \text {. Prepatency was determined by } \\
\text { detecting the presence of GFP }{ }^{+} \text {red blood cells via flow cytometry analysis. The number of mice carrying parasites on } \\
\text { days } 3 \text { ( } 72 \text { hours) and } 5 \text { after sporozoite injection is shown. The total number of infected mice is given in parentheses. } \\
\text { The number of mice alive on day } 10 \text { is also shown. }\end{array}$} \\
\hline
\end{tabular}

under mefloquine or metformin single-drug treatments $(P=0.013$ and $P=0.041$; Figure 3C). Of note, mice under single or combined metformin treatments showed an extended survival, whereas $84 \%$ of the nontreated mice succumbed within 1 week after infection (Table 1). In conclusion, metformin treatment in combination with suboptimal dosage of conventional antimalarials is more efficacious in reducing parasite load.

\section{Discussion}

Metformin is listed by the World Health Organization as an essential medicine to treat and prevent type 2 diabetes. As a biguanide, metformin is related to guanidine and galegine, which can both be extracted from goat's rue (Galega officionalis). This medicinal plant was traditionally used in medieval Europe as a treatment for diabetes and other ailments, including infections such as the plague and typhoid fever. Metformin was first synthesized in 1922, but its use as an antidiabetic agent was only proposed in 1957, a discovery closely linked to malaria owing to the structural relatedness of metformin to proguanil, a highly potent antimalarial biguanide. In the 1940s, metformin was tested in patients with an ongoing malaria blood stage infection while a search was underway for alternatives to proguanil, which was becoming ineffective because of parasite resistance $(34,37)$. Although no clear effect on parasitemia was found (34), lowered glucose levels were later noticed after metformin treatment (37). Indeed, the antihyperglycemia property appears to be common to most biguanides, but metformin outperforms with minimal lactic acidosis.

With a long track record of clinical use, metformin has proved to be highly tolerable, with a strong safety profile and few interactions with other medications. In addition, metformin is water-soluble, is orally bioavailable, has excellent pharmacokinetic adsorption and excretion properties, and has a low cost of production, making it highly affordable and accessible $(37,38)$. These properties contribute to metformin's qualifications as an optimal drug for repurposing (39). Interest in metformin repurposing has been sparked in recent years because of numerous epidemiologic observations and meta-analyses demonstrating positive outcomes and improved prognosis in diabetes patients diagnosed with cancer. To date more than 100 clinical trials in oncology are underway to determine the benefits of metformin as adjunctive therapy in treating and preventing cancer and longevity-related causes, such as inflammation, senescence, and oxidative damage (39-41). It is also being studied as a potential adjunctive therapy to treat infections such as tuberculosis $(25,42)$ and AIDS (43).

The mechanism of action of metformin is not fully defined because it appears to have multiple pleiotropic direct and indirect effects on various cell processes and cell targets. In the treatment of diabetes, metformin is widely used to decrease glucose production and output from the liver and increase circulating insulin sensitivity. These effects are thought to be a result of metformin's interaction with mitochondria, where it alters cellular bioenergetics by inhibiting the respiratory chain complex I. This leads to a decrease in oxidative phosphorylation and subsequent drop in energy with altered ADP-to-ATP ratios $(22,44,45)$. As a consequence, several pathways are affected, such as through the activation of the energy sensor AMPK and a shutdown in gluconeogenesis (46-48). AMPK-dependent processes that are altered include suppression of $\mathrm{mTOR}$ and insulin receptor signaling (IGF-I) via PI3K/AKT pathways, leading to decreased protein synthesis and cell growth and increased apoptosis and autophagy (49). Other mechanisms independent of 
AMPK include direct suppression of regulators such as mTOR at higher metformin concentrations to inhibit hepatic protein synthesis (50), as well as stabilization and induction of p53, which inactivates cyclin D1 and retinoblastoma proteins to inhibit proliferation and promote apoptosis (51-53). Metformin also leads to a reduction in gluconeogenesis and direct inhibition of mitochondrial glycerophosphate dehydrogenase and other mitochondria-dependent biosynthetic pathways, leading to an altered redox state, inhibition of mitochondrial respiration, and availability of macromolecules $(54,55)$.

In the context of suppressed liver stage Plasmodium infection reported in this study, it remains to be determined whether metformin is targeting the host or both the host and the parasite. Potential mechanisms and targets on the host cell include the aforementioned anticancer processes demonstrated to be executed by metformin, such as increased AMPK and p53 signaling and decreased AKT signaling, because these pathways have previously been described to alter infection $(16,17,20,56)$. Of particular interest is the concept that infected hepatocytes undergo a metabolic switch with pro-proliferative tendencies that allow parasite growth $(7,20)$. Consistent with our in vitro and in vivo data of reduced intrahepatic schizont size under metformin treatment, a shutdown of a pro-proliferative program within the host cell would limit parasite growth. In addition to suppression of pro-proliferative pathways during liver stage infection, metformin could also promote cell-autonomous host defense mechanisms, such as increased apoptosis, autophagy, lysosome-vacuole fusion, type I interferon signaling, and reactive oxygen species-mediated killing, all of which have been shown to induce parasite killing and clearance of Plasmodium-infected hepatocytes via infiltrating phagocytes (57-60). Similar metformin-mediated mechanisms have been described for other intracellular pathogens, such as M. tuberculosis (25), Legionella pneumophilia (61), and hepatitis C virus (62).

Metformin has also been shown to mediate immune modulation, such as enhanced $\mathrm{T}$ and B cell effector functions and memory (63-65). Interestingly, a recent study showed that metformin had an effect on the host immunity leading to partial protection of rodents infected with P. yoelii blood stages (35). Although Miyakoda et al. focused on the erythrocytic infection, the effect of metformin on the immune system would be worth exploring within the context of the liver microenvironment, as would a potential role in vaccine development and sterile immunity (66). Future work should also consider a potential metformin-mediated effect on the Plasmodium parasite. The antimalarial proguanil, also a biguanide, works synergistically with atovaquone (another antimalarial) to target the parasite mitochondrial electron transport chain, and when metabolized into cycloguanil (a triazine), directly targets the parasite dihydrofolate reductase enzyme (67). Metformin is not metabolized, but one cannot exclude that unchanged metformin can target the parasite directly. It would be interesting to determine the subcellular localization of metformin, both in infected hepatocytes and erythrocytes, using fluorophores and click chemistry (68). Growth inhibition assays using parasite mutants for potential metformin targets (e.g., mitochondrial components or the AMPK homolog, KIN; ref. 69) may also provide insights into the mechanism by which metformin operates.

In summary, our results provide compelling evidence for the chemopreventive potential of using metformin in the context of malaria infections, whose potential can be enhanced by combination with conventional antimalarials, with the purpose of reducing their dose and associated side effects. Although encouraging, we acknowledge that the doses used in our mice and in vitro assays might not be helpful at predicting the drug concentration required for antimalarial prophylactic activity in humans. This is an unfortunate limitation of using mouse and in vitro models to assess the clinical relevance of metformin $(21,70)$. It remains unclear why rodents require administration of higher metformin doses to reach plasma concentrations comparable to those of patients with diabetes under a standard clinical dose. Nevertheless, the presented data represent a proof of concept for repurposing of an oral agent with an excellent track record for safety and tolerability. Another advantage of metformin as an adjuvant therapy is that it is already approved and a candidate drug already in clinical trials as adjuvant therapy in other infectious diseases, as well as cancer. Thus, the use of metformin warrants future clinical studies to assess efficacy, safety, and potential as a prophylactic antimalarial.

\section{Methods}

Chemicals. Metformin (catalog D150959), Metformin-(dimethyl-d6) hydrochloride (catalog 53183), primaquine bisphosphate (catalog 160393), and mefloquine hydrochloride (catalog M2319) were purchased from MilliporeSigma.

Antibodies. Anti-PbUIS4 goat polyclonal was purchased from SICGEN (AB0042-200). Anti-PfHSP70 mouse monoclonal (4C9) was a gift from Fidel Zavala (John Hopkins University, Baltimore, Maryland, USA). 
Parasite lines. Transgenic P. berghei ANKA parasites expressing GFP (259c12) and GFP/luciferase (676m1c11) were obtained from the Leiden Malaria Research Group (www.pberghei.eu). Transgenic P. berghei ANKA sporozoites were isolated from salivary glands of Anopheles stephensi mosquitoes bred at Instituto de Medicina Molecular João Lobo Antunes (Lisboa, Portugal). P. falciparum NF54-infected Anopheles stephensi mosquitoes were obtained from John Hopkins University Malaria Research Institute (Baltimore, Maryland, USA) or Sanaria Inc (Rockville, Maryland, USA). Cryostabilates of P. falciparum 3D7-infected erythrocytes were obtained through the MR4 (https://www.beiresources.org/About/MR4.aspx).

Animals, drug treatments, and infections. Male C57BL/6 mice 6-8 weeks old were purchased from Charles River Laboratories, Inc, and housed in specific pathogen-free facilities of the Instituto de Medicina Molecular João Lobo Antunes (Lisboa, Portugal). Mice were infected with freshly dissected P. berghei sporozoites by intravenous injection, with the inoculum of 500 or 10,000 as indicated in the corresponding figure legend. For all drug treatments, mice were weighed to determine appropriate dosage, which was adjusted accordingly. Metformin was administered prophylactically starting at day -7 via drinking water to a final dose of $500 \mathrm{mg} / \mathrm{kg} / \mathrm{d}(\sim 2.5 \mathrm{mg} / \mathrm{mL}$ as determined by mouse weight and daily water intake volume per mouse). Primaquine was dissolved in $0.9 \% \mathrm{NaCl}$ at a dose of $15 \mathrm{mg} / \mathrm{kg}$ in a total volume of 200 $\mu \mathrm{L}$ and administered as a single dose at 2 hours after sporozoite inoculation by intraperitoneal injection. The suboptimal dose of primaquine was determined based on previous studies (71, 72). Mefloquine was dissolved $1 \%$ methanol $(v / v)$ in $0.9 \% \mathrm{NaCl}$ in a total volume of $400 \mu \mathrm{L}$ and injected by intraperitoneal injection daily starting on the day of sporozoite inoculation and up to day 5 after infection (73).

Cell culture and infection. Primary mouse hepatocytes were isolated using a 2-step in situ perfusion method, as described previously (20). Percoll-purified hepatocytes were seeded in collagen-coated 96-well plates and infected the day after with freshly dissected $P$. berghei sporozoites (8000/well). Metformin was added 2 hours after infection in increasing concentrations (in triplicate) for $\mathrm{IC}_{50}$ determination to generate an 8-point dose response. $\mathrm{IC}_{50}$ values were calculated by fitting the data to a log dose-response curve using GraphPad (Prism).

Cryopreserved primary human hepatocytes purchased from BioIVT were seeded on collagen-micropatterned 96-well plates, as described previously $(29,32)$. Hepatocytes were infected with freshly dissected P. falciparum sporozoites (60,000/well), and metformin was added 3 hours later at 50- and 200- $\mu$ M final concentrations.

$P$. falciparum blood cultures were maintained in RPMI supplemented with $25 \mathrm{mM}$ HEPES, $100 \mu \mathrm{M}$ hypoxanthine, $2 \mathrm{mM}$ L-glutamine, $50 \mu \mathrm{g} / \mathrm{L}$ gentamicin, and $0.5 \%$ albumax II and $2 \%$ hematocrit. The in vitro drug assay was performed in 96 -well plates. Metformin was added in duplicate with a series of 2-fold dilutions, and starting parasitemia was adjusted to $0.1 \%$. Parasitemia was measured by flow cytometry at 48 hours after drug exposure, and $\mathrm{IC}_{50}$ values were determined as described above.

Luminescence and cell viability assays. Primary mouse hepatocytes were washed with PBS at 48 hours after infection and lysed for 20 minutes in $75 \mu \mathrm{L}$ of lysis buffer from Firefly Luciferase Assay Kit (Biotium). Fifty microliters of D-Luciferin dissolved in the kit buffer was added to $30 \mu \mathrm{L}$ of total lysate in white 96 -well plates, and luminescence was measured using a multiplate reader (Infinite 200M, Tecan). Cell viability was assayed by CellTiter Blue cell viability assay (Promega) using the manufacturer's protocol, following an incubation of 90 minutes at $37^{\circ} \mathrm{C}$, and fluorescence was measured with a multiplate reader (Infinite 200M, Tecan).

Immunofluorescence assay. The median lobes of livers were fixed with $4 \%$ paraformaldehyde for 2 hours and sliced into 50- $\mu \mathrm{m}$ thick sections using the Vibratome VT $1000 \mathrm{~S}$ (Leica). To detect $P$. berghei parasites, liver sections were permeabilized and blocked in $1 \%$ BSA and $0.3 \%$ Triton X-100 for 45 minutes and incubated with goat anti-PbUIS4 antibodies for 1 hour at room temperature. After washing, liver sections were incubated with anti-goat conjugated to Alexa Fluor 555 (Thermo Fisher Scientific, catalog A21432), anti-GFP conjugated to Alexa Fluor 488 (Thermo Fisher Scientific, catalog A21311), and Hoechst (Thermo Fisher Scientific, catalog 33258) for another 1 hour at room temperature. Hoechst 33258 (H3569) was obtained from Thermo Fisher Scientific. Finally, 6-8 sections representing an equal distribution of lobe per mouse were mounted in Fluoromount (Southern Biotech) and imaged on an Axiovert 200M (Zeiss). For analysis, the entire section was scanned, and images of parasites were recorded. Parasite area was quantified with ImageJ software (NIH) based on GFP and PbUIS4 staining.

Primary human hepatocytes were fixed in cold methanol for 10 minutes and stored at $4^{\circ} \mathrm{C}$ after PBS washing. To detect $P$. falciparum parasites, cells were blocked in $2 \%$ BSA for 30 minutes and incubated with anti-PfHSP70 monoclonal antibodies (4C9) for 1 hour at room temperature. Cells were then washed in PBS and incubated with anti-mouse conjugated to Alexa Fluor 594 (Thermo Fisher Scientific, A-21203) 
for 1 hour at room temperature. Next, cells were counterstained with Hoechst and mounted in Aquamount (Thermo Scientific, catalog 41799-008). Images of all parasites in the well were captured on a Nikon Eclipse Ti microscope and analyzed with ImageJ.

Flow cytometry. A drop $(2-3 \mu \mathrm{L})$ of blood was collected from the mouse tail, placed into $500 \mu \mathrm{L}$ PBS, and analyzed in an LSR Fortessa flow cytometer (BD Biosciences). Per mouse, 1-2 million events were acquired at the indicated time points. BD FACSDiva software was used for data acquisition and FlowJo software for further analysis. Cells were selected on the basis of their size by gating first on FSC and SSC and, subsequently, plotted on FITC (green) and PE (red) channels to eliminate autofluorescent events. The GFP-expressing parasites were detected in the FITC channel.

Metformin quantification. Naive mice, treated with $500 \mathrm{mg} / \mathrm{kg}$ in drinking water as described above for 7 days, were bled by heart puncture for plasma preparation into K2EDTA tubes. Plasma samples were stored at $-80^{\circ} \mathrm{C}$ until analysis at Mass Spectrometry Unit, Instituto de Tecnologia Química e Biologia/Instituto de Biologia Experimental e Tecnológica (Oeiras, Portugal). Briefly, metformin and metformin-D6 standard solutions were prepared in $50 \%$ acetonitrile/water. Samples were prepared by adding $47.5 \%$ acetonitrile and $2.5 \mu \mathrm{M}$ metformin-D6 to $40 \mu \mathrm{L}$ of plasma, followed by centrifugation at $18,000 \mathrm{~g}$ for 10 minutes at $1^{\circ} \mathrm{C}$. A standard calibration curve was prepared similarly to the samples, with a series of 2-fold dilutions of metformin spiked in $20 \mu \mathrm{L}$ of nontreated plasma. Sample and standard injections $(1 \mu \mathrm{L})$ were performed in triplicate. Chromatographic analysis was performed on an Ultimate 3000 UHPLC and the separation on a Waters XBridge column C18 $(2.1 \times 150 \mathrm{~mm}, 3.5-\mu \mathrm{m}$ particle size). The mobile phases A and B were water and acetonitrile with $0.1 \%$ formic acid, respectively. Column temperature was maintained at $30^{\circ} \mathrm{C}$ and flow rate at $400 \mu \mathrm{L} / \mathrm{min}$. The data were acquired on the Q Exactive Focus coupled to UHPLC, using Xcalibur software v.4.0.27.19.

Statistics. Statistics were determined with Student's $t$ (2 tailed) and Mann-Whitney $U$ tests for comparisons between 2 conditions and 1-way ANOVA for comparisons involving 3 or more conditions. All samples were included in the analysis. $P$ values below 0.05 were considered statistically significant. Data mentioned in the text are represented as mean $\pm \mathrm{SD}$.

Study approval. Experimental procedures were approved by the internal Animal Ethics Committee and performed in accordance with the Portuguese law for the use of laboratory animals (Decree-Law 113/2013), the European Directive 2010/63/EU, and the Federation of European Laboratory Animal Science Associations guidelines and recommendations concerning laboratory animal welfare. The primary human hepatocytes used in this study are commercially available. Approvals and certificates were obtained by the vendors.

\section{Author contributions}

IMV, MTGR, LFLR, SM, and LMS performed the experiments and analyzed the results. IMV and LMS wrote the manuscript. SNB, MMM, and LMS supervised the work.

\section{Acknowledgments}

We would like to thank Daniel Carapau, David Cristina, and Jeremy Burrows for thoughtful discussions; Ana Parreira for mosquito and sporozoite production; Vanessa Zuzarte-Luís, Maria Rebelo, Alex Miller, and Sandra March for technical assistance and advice; Eliana Real for critical reading of the manuscript; and Patrick Durkin for proofreading the text. This work was supported by Fundação para a Ciência e Tecnologia (Portugal) PTDC/SAU-MET/118199/2010 to LMS and European Research Council Proof of Concept Grant to MMM (ERC-2015-PoC-DL3-713691-REUSE4MALARIA). SNB is a Howard Hughes Medical Institute Investigator.

Address correspondence to: Maria M. Mota and Liliana Mancio-Silva, Instituto de Medicina Molecular João Lobo Antunes, Faculdade de Medicina da Universidade de Lisboa, Avenida Professor Egas Moniz, 1649-028 Lisboa, Portugal. Phone: 351.217.999.411; Email: mmota@medicina.ulisboa.pt (MMM); liliana.mancio@inserm.fr (LMS).

ISV's present address is: Department of Internal Medicine, Division of Infectious Disease, University of South Florida, Morsani College of Medicine, Tampa, Florida, USA.

MTGR's present address is: Unité Mixte de Recherche 5235, Centre National de la Recherche Scientifique, Université de Montpellier 2, Montpellier, France. 
LFLR's present address is: Laboratory of Molecular Microbiology, Global Health Institute, School of Life Sciences, École Polytechnique Fédérale de Lausanne, Lausanne, Switzerland.

LM-S's present address is: Institut National de la Santé et de la Recherche Médicale, Unité 1201, Paris, France.

1. World Health Organization. World Malaria Report 2010. http://www.who.int/malaria/world_malaria_report_2010/en/index. html. Accessed November 18, 2019.

2. Vaughan AM, Kappe SHI. Malaria parasite liver infection and exoerythrocytic biology. Cold Spring Harb Perspect Med. 2017;7(6):a025486.

3. Holz LE, Fernandez-Ruiz D, Heath WR. Protective immunity to liver-stage malaria. Clin Transl Immunology. 2016;5(10):e105.

4. Hooft van Huijsduijnen R, Wells TN. The antimalarial pipeline. Curr Opin Pharmacol. 2018;42:1-6.

5. Watson J, Taylor WRJ, Bancone G, Chu CS, Jittamala P, White NJ. Implications of current therapeutic restrictions for primaquine and tafenoquine in the radical cure of vivax malaria. PLoS Negl Trop Dis. 2018;12(4):e0006440.

6. Mathews ES, Odom John AR. Tackling resistance: emerging antimalarials and new parasite targets in the era of elimination. F1000Res. 2018;7:F1000 Faculty Rev-1170.

7. Glennon EKK, Dankwa S, Smith JD, Kaushansky A. Opportunities for host-targeted therapies for malaria. Trends Parasitol. 2018;34(10):843-860.

8. Prudêncio M, Mota MM. Targeting host factors to circumvent anti-malarial drug resistance. Curr Pharm Des. 2013;19(2):290-299.

9. Langhorne J, Duffy PE. Expanding the antimalarial toolkit: targeting host-parasite interactions. J Exp Med. 2016;213(2):143-153.

10. Yadav DK, Kumar S, Teli MK, Yadav R, Chaudhary S. Molecular targets for malarial chemotherapy: a review. Curr Top Med Chem. 2019;19(10):861-873

11. Murphy SC, Harrison T, Hamm HE, Lomasney JW, Mohandas N, Haldar K. Erythrocyte G protein as a novel target for malarial chemotherapy. PLoS Med. 2006;3(12):e528.

12. Pamplona A, et al. Heme oxygenase-1 and carbon monoxide suppress the pathogenesis of experimental cerebral malaria. Nat Med. 2007;13(6):703-710.

13. Pena AC, et al. A novel carbon monoxide-releasing molecule fully protects mice from severe malaria. Antimicrob Agents Chemother. 2012;56(3):1281-1290.

14. Silvie O, et al. Hepatocyte CD81 is required for Plasmodium falciparum and Plasmodium yoelii sporozoite infectivity. Nat Med. 2003;9(1):93-96.

15. Foquet L, et al. Anti-CD81 but not anti-SR-BI blocks Plasmodium falciparum liver infection in a humanized mouse model. J Antimicrob Chemother. 2015;70(6):1784-1787.

16. Kaushansky A, et al. Suppression of host p53 is critical for Plasmodium liver-stage infection. Cell Rep. 2013;3(3):630-637.

17. Douglass AN, et al. Host-based prophylaxis successfully targets liver stage malaria parasites. Mol Ther. 2015;23(5):857-865.

18. Arang N, et al. Identifying host regulators and inhibitors of liver stage malaria infection using kinase activity profiles. Nat Commun. 2017;8(1):1232.

19. Prudêncio M, et al. Kinome-wide RNAi screen implicates at least 5 host hepatocyte kinases in Plasmodium sporozoite infection. PLoS Pathog. 2008;4(11):e1000201.

20. Ruivo MTG, et al. Host AMPK is a modulator of Plasmodium liver infection. Cell Rep. 2016;16(10):2539-2545.

21. Chandel NS, et al. Are metformin doses used in murine cancer models clinically relevant? Cell Metab. 2016;23(4):569-570.

22. Foretz M, et al. Metformin inhibits hepatic gluconeogenesis in mice independently of the LKB1/AMPK pathway via a decrease in hepatic energy state. J Clin Invest. 2010;120(7):2355-2369.

23. Heise M, et al. Downregulation of organic cation transporters OCT1 (SLC22A1) and OCT3 (SLC22A3) in human hepatocellular carcinoma and their prognostic significance. BMC Cancer. 2012;12:109.

24. Jouan E, Le Vée M, Denizot C, Parmentier Y, Fardel O. Drug transporter expression and activity in human hepatoma HuH-7 cells. Pharmaceutics. 2016;9(1):E3.

25. Singhal A, et al. Metformin as adjunct antituberculosis therapy. Sci Transl Med. 2014;6(263):263ra159.

26. Graham GG, et al. Clinical pharmacokinetics of metformin. Clin Pharmacokinet. 2011;50(2):81-98.

27. Kebaier C, Voza T, Vanderberg J. Kinetics of mosquito-injected Plasmodium sporozoites in mice: fewer sporozoites are injected into sporozoite-immunized mice. PLoS Pathog. 2009;5(4):e1000399.

28. Medica DL, Sinnis P. Quantitative dynamics of Plasmodium yoelii sporozoite transmission by infected anopheline mosquitoes. Infect Immun. 2005;73(7):4363-4369.

29. March S, et al. Micropatterned coculture of primary human hepatocytes and supportive cells for the study of hepatotropic pathogens. Nat Protoc. 2015;10(12):2027-2053.

30. March S, et al. A microscale human liver platform that supports the hepatic stages of Plasmodium falciparum and vivax. Cell Host Microbe. 2013;14(1):104-115.

31. Gural N, Mancio-Silva L, He J, Bhatia SN. Engineered livers for infectious diseases. Cell Mol Gastroenterol Hepatol. 2018;5(2):131-144.

32. Khetani SR, Bhatia SN. Microscale culture of human liver cells for drug development. Nat Biotechnol. 2008;26(1):120-126.

33. Saisho Y. Metformin and inflammation: its potential beyond glucose-lowering effect. Endocr Metab Immune Disord Drug Targets. 2015;15(3):196-205

34. Garcia EY. Flumamine, a new synthetic analgesic and anti-flu drug. J Philipp Med Assoc. 1950;26(7):287-293.

35. Miyakoda M, Bayarsaikhan G, Kimura D, Akbari M, Udono H, Yui K. Metformin promotes the protection of mice infected with Plasmodium yoelii independently of $\gamma \delta \mathrm{T}$ cell expansion. Front Immunol. 2018;9:2942.

36. Nevin RL, Byrd AM. Neuropsychiatric adverse reactions to mefloquine: a systematic comparison of prescribing and patient safety guidance in the US, UK, Ireland, Australia, New Zealand, and Canada. Neurol Ther. 2016;5(1):69-83. 
37. Bailey CJ. Metformin: historical overview. Diabetologia. 2017;60(9):1566-1576.

38. Foretz M, Guigas B, Bertrand L, Pollak M, Viollet B. Metformin: from mechanisms of action to therapies. Cell Metab. 2014;20(6):953-966.

39. Heckman-Stoddard BM, DeCensi A, Sahasrabuddhe VV, Ford LG. Repurposing metformin for the prevention of cancer and cancer recurrence. Diabetologia. 2017;60(9):1639-1647.

40. Barzilai N, Crandall JP, Kritchevsky SB, Espeland MA. Metformin as a tool to target aging. Cell Metab. 2016;23(6):1060-1065

41. Chae YK, et al. Repurposing metformin for cancer treatment: current clinical studies. Oncotarget. 2016;7(26):40767-40780.

42. Malik F, et al. Is metformin poised for a second career as an antimicrobial? Diabetes Metab Res Rev. 2018;34(4):e2975.

43. Lamarca K, García Sarasola A, Vidal F, Domingo P. Drug therapies for HIV-related metabolic disorders. Expert Opin Pharmacother. 2016;17(10):1327-1338.

44. Owen MR, Doran E, Halestrap AP. Evidence that metformin exerts its anti-diabetic effects through inhibition of complex 1 of the mitochondrial respiratory chain. Biochem J. 2000;348 Pt 3:607-614.

45. El-Mir MY, Nogueira V, Fontaine E, Avéret N, Rigoulet M, Leverve X. Dimethylbiguanide inhibits cell respiration via an indirect effect targeted on the respiratory chain complex I. J Biol Chem. 2000;275(1):223-228.

46. Cao J, et al. Low concentrations of metformin suppress glucose production in hepatocytes through AMP-activated protein kinase (AMPK). J Biol Chem. 2014;289(30):20435-20446.

47. Shaw RJ, et al. The kinase LKB1 mediates glucose homeostasis in liver and therapeutic effects of metformin. Science. 2005;310(5754):1642-1646.

48. Zhou G, et al. Role of AMP-activated protein kinase in mechanism of metformin action. J Clin Invest. 2001;108(8):1167-1174.

49. Morales DR, Morris AD. Metformin in cancer treatment and prevention. Annu Rev Med. 2015;66:17-29.

50. Howell JJ, et al. Metformin inhibits hepatic mTORC1 signaling via dose-dependent mechanisms involving AMPK and the TSC complex. Cell Metab. 2017;25(2):463-471.

51. Queiroz EA, et al. Metformin induces apoptosis and cell cycle arrest mediated by oxidative stress, AMPK and FOXO3a in MCF-7 breast cancer cells. PLoS ONE. 2014;9(5):e98207.

52. He G, et al. AMP-activated protein kinase induces p53 by phosphorylating MDMX and inhibiting its activity. Mol Cell Biol. 2014;34(2):148-157.

53. Ben Sahra I, et al. Metformin, independent of AMPK, induces mTOR inhibition and cell-cycle arrest through REDD1. Cancer Res. 2011;71(13):4366-4372.

54. Madiraju AK, et al. Metformin suppresses gluconeogenesis by inhibiting mitochondrial glycerophosphate dehydrogenase Nature. 2014;510(7506):542-546.

55. Griss T, et al. Metformin antagonizes cancer cell proliferation by suppressing mitochondrial-dependent biosynthesis. PLoS Biol. 2015;13(12):e1002309.

56. van de Sand C, et al. The liver stage of Plasmodium berghei inhibits host cell apoptosis. Mol Microbiol. 2005;58(3):731-742.

57. Real E, et al. Plasmodium UIS3 sequesters host LC3 to avoid elimination by autophagy in hepatocytes. Nat Microbiol. 2018;3(1):17-25.

58. Epiphanio S, et al. Heme oxygenase-1 is an anti-inflammatory host factor that promotes murine plasmodium liver infection. Cell Host Microbe. 2008;3(5):331-338.

59. Zuzarte-Luís V, et al. Dietary alterations modulate susceptibility to Plasmodium infection. Nat Microbiol. 2017;2(12):1600-1607.

60. Liehl P, et al. Host-cell sensors for Plasmodium activate innate immunity against liver-stage infection. Nat Med. 2014;20(1):47-53.

61. Kajiwara C, et al. Metformin mediates protection against Legionella pneumonia through activation of AMPK and mitochondrial reactive oxygen species. J Immunol. 2018;200(2):623-631.

62. Nakashima K, Takeuchi K, Chihara K, Hotta H, Sada K. Inhibition of hepatitis C virus replication through adenosine monophosphate-activated protein kinase-dependent and -independent pathways. Microbiol Immunol. 2011;55(11):774-782

63. Eikawa S, Nishida M, Mizukami S, Yamazaki C, Nakayama E, Udono H. Immune-mediated antitumor effect by type 2 diabetes drug, metformin. Proc Natl Acad Sci USA. 2015;112(6):1809-1814.

64. Pearce EL, et al. Enhancing CD8 T-cell memory by modulating fatty acid metabolism. Nature. 2009;460(7251):103-107.

65. Diaz A, Romero M, Vazquez T, Lechner S, Blomberg BB, Frasca D. Metformin improves in vivo and in vitro B cell function in individuals with obesity and Type-2 Diabetes. Vaccine. 2017;35(20):2694-2700.

66. Frevert U, Krzych U. Plasmodium cellular effector mechanisms and the hepatic microenvironment. Front Microbiol. 2015;6:482.

67. Srivastava IK, Vaidya AB. A mechanism for the synergistic antimalarial action of atovaquone and proguanil. Antimicrob Agents Chemother. 1999;43(6):1334-1339.

68. Müller S, et al. Metformin reveals a mitochondrial copper addiction of mesenchymal cancer cells. PLoS ONE. 2018;13(11):e0206764.

69. Mancio-Silva L, et al. Nutrient sensing modulates malaria parasite virulence. Nature. 2017;547(7662):213-216.

70. Dowling RJ, et al. Metformin pharmacokinetics in mouse tumors: implications for human therapy. Cell Metab. 2016;23(4):567-568.

71. Mendes AM, Albuquerque IS, Machado M, Pissarra J, Meireles P, Prudêncio M. Inhibition of Plasmodium liver infection by ivermectin. Antimicrob Agents Chemother. 2017;61(2):e02005-16.

72. Ploemen $\mathrm{IH}$, et al. Visualisation and quantitative analysis of the rodent malaria liver stage by real time imaging. PLoS ONE. 2009;4(11):e7881.

73. Souraud JB, et al. Atorvastatin treatment is effective when used in combination with mefloquine in an experimental cerebral malaria murine model. Malar J. 2012;11:13. 